\title{
Intrinsic origin of interfacial second-order magnetic anisotropy in ferromagnet/normal metal heterostructures
}

\author{
Hyung Keun Gweon', Hyeon-Jong Park², Kyoung-Whan Kim $\mathbb{1}^{3}$, Kyung-Jin Lee $\mathbb{B}^{1,2}$ and Sang Ho Lim (D)
}

\begin{abstract}
Interfacial perpendicular magnetic anisotropy, which is characterized by first-order $\left(K_{1}\right)$ and second-order $\left(K_{2}\right)$ anisotropy, is the core phenomenon for nonvolatile magnetic devices. A sizable $K_{2}$ satisfying a specific condition stabilizes the easy-cone state, where equilibrium magnetization forms at an angle from the film normal. The easy-cone state offers intriguing possibilities for advanced spintronic devices and unique spin textures, such as spin superfluids and easy-cone domain walls. Experimental realization of the easy-cone state requires understanding the origin of $K_{2}$, thereby enhancing $K_{2}$. However, the previously proposed origins of $K_{2}$ cannot fully account for the experimental results. Here, we experimentally show that $K_{2}$ scales almost linearly with the work function difference between the Co and $\mathrm{X}$ layers in $\mathrm{Pt} / \mathrm{Co} / \mathrm{X}$ heterostructures $(\mathrm{X}=\mathrm{Pd}, \mathrm{Cu}, \mathrm{Pt}, \mathrm{Mo}, \mathrm{Ru}, \mathrm{W}$, and Ta), suggesting the central role of the inversion asymmetry in $K_{2}$. Our result provides a guideline for enhancing $K_{2}$ and realizing magnetic applications based on the easy-cone state.
\end{abstract}

\section{Introduction}

Magnetic anisotropy describes a magnetization-angledependent change in magnetic energy and stabilizes the magnetization in specific directions. Its angular dependence is determined by the symmetry of the crystal or structure. In thin-film heterostructures such as ferromagnet/normal metal bilayers where the structural inversion symmetry is broken at the interface, the magnetic anisotropy is dominated by interfacial contributions, as follows (up to the second order):

$$
E(\theta)=K_{1}^{\mathrm{eff}} \sin ^{2} \theta+K_{2} \sin ^{4} \theta
$$

where $K_{1}^{\text {eff }}\left(=K_{1}-2 \pi M_{\mathrm{s}}^{2}\right)$ is the effective first-order anisotropy energy density that comprises the demagnetization

\footnotetext{
Correspondence: Kyung-Jin Lee (kj_lee@korea.ac.kr) or

Sang Ho Lim (sangholim@korea.ac.kr)

'Department of Materials Science and Engineering, Korea University, Seoul 02841, Korea

${ }^{2}$ KU-KIST Graduate School of Converging Science and Technology, Korea University, Seoul 02841, Korea

Full list of author information is available at the end of the article
}

energy density (with $K_{1}$ and $M_{\mathrm{s}}$ being the first-order anisotropy energy density and saturation magnetization, respectively), $K_{2}$ is the second-order anisotropy energy density, and $\theta$ is the polar angle of the magnetization. The magnetic phase diagram as functions of $K_{1}^{\text {eff }}$ and $K_{2}$ (Fig. 1a) shows several distinct magnetic states ${ }^{1}$. Among them, the out-of-plane state originating from perpendicular magnetic anisotropy (PMA) has been a main focus of spintronics research ${ }^{2}$ because it offers scalable magnetic random-access memories (MRAMs) ${ }^{3}$.

Recently, interest in another state-the easy-cone state, where the equilibrium magnetization direction is tilted from the film normal and forms a cone-has increased for the following reasons. It provides improved functionalities of various spintronics devices, such as low-power operation of spin-transfer torque (STT) MRAMs ${ }^{4-6}$ and zerofield precession of STT oscillators ${ }^{7}$. Moreover, it hosts spin superfluids associated with spontaneous breaking of $U(1)$ spin-rotational symmetry ${ }^{8,9}$ and allows unique easycone domain wall dynamics ${ }^{10}$. The existence of the easycone state was experimentally verified in various layered 

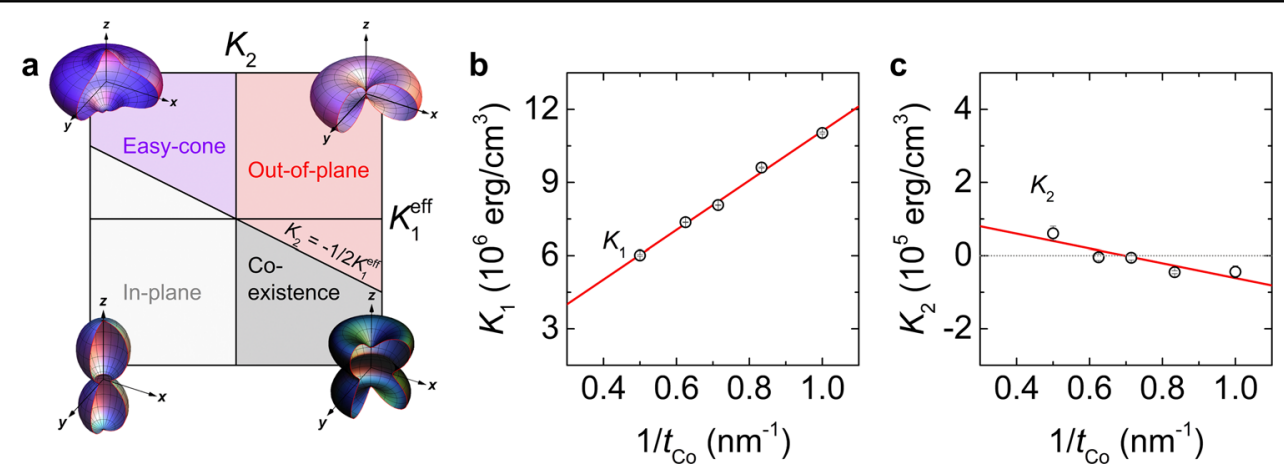

Fig. 1 Phase diagram showing various magnetic states and inverse thickness dependences of $K_{\mathbf{1}}$ and $\boldsymbol{K}_{\mathbf{2}}$. a Magnetic phase diagram as functions of $K_{1}^{\text {eff }}$ and $K_{2}$, showing four different magnetic states and their energy surfaces. $\mathbf{b}, \mathbf{c}$ Inverse Co thickness dependence of $K_{1}(\mathbf{b})$ and $K_{2}(\mathbf{c})$ for the $\mathrm{Pt} / \mathrm{Co}\left(t_{\mathrm{co}}\right) / \mathrm{Cu}$ structure. The error bars in $K_{1}$ and $K_{2}$ were obtained from three repeated measurements. The negative slope in (c) indicates negative interfacial $K_{2}$.

structures $^{6,11,12}$. However, the design window for forming a stable easy-cone state is very narrow ${ }^{6,11,12}$, which presents a critical challenge for realizing magnetic devices utilizing the easy-cone state.

In contrast to the out-of-plane state that can form with $K_{1}$ alone, the easy-cone state requires a large $K_{2}$ value; it is formed for $K_{1}^{\text {eff }}<0$ and $K_{2}>-1 / 2 K_{1}^{\text {eff }}$ (Fig. 1a). To actively employ the easy-cone state in various applications, therefore, it is of crucial importance to find a way of enhancing $K_{2}$, which necessitates a fundamental understanding of its origin. The origin of $K_{1}$ has long been a subject of extensive theoretical and experimental research. It was found to depend on the orbital anisotropy $^{13}$, spin-orbit interaction of electronic structures near the Fermi level ${ }^{14}$ or Rashba-type spin-orbit interaction at the interface associated with the inversion symmetry breaking ${ }^{15-17}$. Concerning the origin of $K_{2}$, three mechanisms have been proposed: (1) spatial fluctuations of $K_{1}{ }^{18}$, (2) interfacial PMA combined with a gradual weakening of the exchange energy along the thickness direction ${ }^{19}$, and (3) the mixture of bulk magnetocrystalline cubic anisotropy and interfacial uniaxial anisotropy ${ }^{20}$. The first and second mechanisms predict only positive $K_{2}$ and fail to explain the negative $K_{2}$ observed in experiments ${ }^{21,22}$. The third mechanism predicts both signs of $K_{2}$ depending on the nature of the bulk cubic anisotropy. Our measurement of $K_{2}$ for a $\mathrm{Pt} / \mathrm{Co} / \mathrm{Cu}$ structure, however, shows that $K_{2}$ is inversely proportional to the Co thickness (thus, the interface origin) and is negative for thin Co layers (see Fig. 1c and Supplementary Note 1 for details). As the third mechanism cannot account for the origin of the negative $K_{2}$ of the interface, none of the three aforementioned mechanisms can explain this experimental observation; thus, a new origin of $K_{2}$ must be identified.

In this study, we focus on the role of the inversion symmetry breaking in $K_{2}$ for the following two reasons.
First, recent theoretical and experimental studies indicated the important role of the inversion asymmetry in $K_{1}$ for ferromagnet-normal metal heterostructures ${ }^{15-17}$. As $K_{1}$ and $K_{2}$ are the order-expanded coefficients of the net magnetic anisotropy [Eq. (1)], it is reasonable to expect that they share the same origin. Second, our measurements of $K_{1}$ and $K_{2}$ for $\mathrm{Pt} / \mathrm{Co} / \mathrm{Cu}$ and $\mathrm{Pt} / \mathrm{Co} / \mathrm{MgO}$ stacks over a wide range of Co thickness $\left(t_{\mathrm{Co}}\right)$ show that for both $K_{1}$ and $K_{2}$, the interfacial contribution is dominant compared with the bulk contribution (Supplementary Note 1), indicating the important role of the inversion asymmetry at the interface in the anisotropy.

\section{Materials and methods}

Sample preparation

To investigate the correlation between the inversion asymmetry and $K_{2}$, we examine various sputtered $\mathrm{Pt} / \mathrm{Co} / \mathrm{X}$ stacks, with $\mathrm{X}=\mathrm{Pd}, \mathrm{Cu}, \mathrm{Pt}, \mathrm{Mo}, \mathrm{Ru}, \mathrm{W}$, and Ta. The stacks investigated in this study had the structure of $\mathrm{Si}$ substrate (wet-oxidized)/Ta $(5 \mathrm{~nm}) / \mathrm{Pt}$ $(5 \mathrm{~nm}) / \mathrm{Co}(1 \mathrm{~nm}) / \mathrm{X}(3 \mathrm{~nm}) / \mathrm{Ta}(3 \mathrm{~nm})$ and were fabricated using an ultrahigh-vacuum magnetron sputtering system with a base pressure of $8 \times 10^{-8}$ Torr. All metallic layers were deposited under an Ar pressure of $2 \times 10^{-3}$ Torr. The Ta under- and upper-layers were introduced to improve the surface roughness and prevent the oxidation of the stacks, respectively. For $\mathrm{X}=$ $\mathrm{Ta}, \mathrm{Pt}(3 \mathrm{~nm})$ was used as the upper layer. $\mathrm{Pt} / \mathrm{Co} / \mathrm{MgO}$ $(2 \mathrm{~nm})$ stacks were also prepared, followed by postannealing at $400{ }^{\circ} \mathrm{C}$ for $30 \mathrm{~min}$ to maximize the interfacial PMA at the $\mathrm{Co} / \mathrm{MgO}$ interface ${ }^{23-25}$. Details regarding the fabrication and annealing are provided in Supplementary Note 5 . The continuous samples were patterned into a Hall bar structure via photolithography and inductively coupled plasma etching. The currentinjection line and the voltage branch had dimensions of $5 \mu \mathrm{m}$ (width) $\times 35 \mu \mathrm{m}$ (length). A 50-nm-thick Pt layer was 

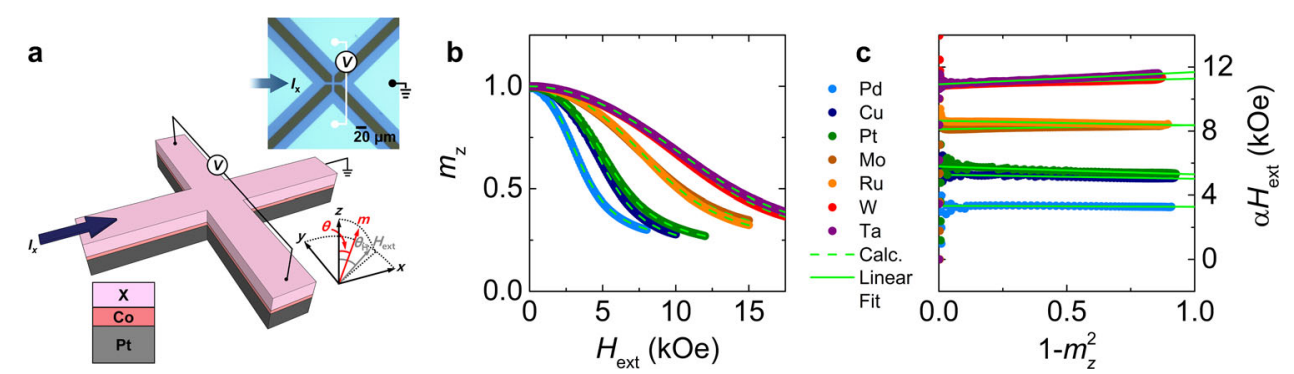

Fig. 2 Measurement of magnetic anisotropy. a Schematic showing the Hall bar device used for the magnetic anisotropy measurements, together with an optical microscopy image (upper right). The Hall voltage was measured while injecting an in-plane current $\left(I_{x}\right)$ along the $x$ direction. The $H_{\text {ext }}$ was applied along $\theta_{H}=80^{\circ}$ to facilitate coherent magnetization behavior. $\mathbf{b} m_{z}-H_{\text {ext }}$ plots for $\mathrm{Pt} / \mathrm{Co} / \mathrm{X}$ heterostructures. The symbols and dashed lines indicate the results of AHE measurements and macrospin simulations, respectively. $\mathbf{c} a H_{\text {ext }} \mathrm{vs} .1-m_{z}^{2}$ plots converted from the results in (b). The solid lines represent the linear fittings to the data.

deposited on top of the patterned structure as a contact pad for magnetotransport characterization (Fig. 2a).

\section{Measurement of magnetic anisotropy}

The magnetic anisotropies $\left(K_{1}\right.$ and $\left.K_{2}\right)$ were characterized by the anomalous Hall effect (AHE) in a standard fourprobe Hall geometry. The Hall bar device was mounted on a rotatable sample stage placed in the gap of an electromagnet. The AHE measurements involved injecting an inplane current $\left(I_{x}=5 \mathrm{~mA}\right)$ along the $x$ direction and sensing the Hall voltage induced along the $y$ direction. The external magnetic field $\left(H_{\text {ext }}\right)$ was applied at a polar angle $\left(\theta_{\mathrm{H}}\right)$ of $80^{\circ}$ to facilitate coherent magnetization behavior (Fig. 2a). The generalized Sucksmith-Thompson method was used to accurately determine the effective first- and second-order anisotropy fields (denoted as $H_{\mathrm{K} 1}^{\text {eff }}=2 K_{1} / M_{\mathrm{s}}-4 \pi M_{\mathrm{s}}$ and $H_{\mathrm{K} 2}=4 K_{2} / M_{\mathrm{s}}$, respectively $)^{26}$. The key to this method is the use of the following equations, which can be derived from the total magnetic energy equation [Eq. (1), considering the Zeeman energy $\left.\left(-\mathbf{M} \cdot \mathbf{H}_{\text {ext }}\right)\right]$ :

$$
\begin{aligned}
& \alpha H_{\mathrm{ext}}=H_{\mathrm{K} 1}^{\mathrm{eff}}+H_{\mathrm{K} 2}\left(1-m_{z}^{2}\right), \\
& \alpha \equiv \frac{m_{z} \sin \theta_{\mathrm{H}}-\sqrt{1-m_{z}^{2}} \cos \theta_{\mathrm{H}}}{m_{z} \sqrt{1-m_{z}^{2}}} .
\end{aligned}
$$

The AHE results were normalized with respect to the anomalous Hall voltages to obtain $m_{z}-H_{\text {ext }}$ curves (Fig. 2b), and then $\alpha H_{\text {ext }}$ was plotted with respect to $1-m_{z}^{2}$ to extract $H_{\mathrm{K} 1}^{\text {eff }}$ and $H_{\mathrm{K} 2}$ from the intercept and slope, respectively [Eq. (2) and Fig. 2c]. We observed a slight misalignment in $\theta_{\mathrm{H}}$ from its nominal value (mostly within $2^{\circ}$ ), which was adjusted to maximize the linearity of the $\alpha H_{\text {ext }}$ vs. $1-m_{z}^{2}$ plot. To confirm the accuracy of the anisotropy constants, the measured $m_{z}-H_{\text {ext }}$ curves were compared with those from macrospin simulations using the obtained $H_{\mathrm{K} 1}^{\text {eff }}$ and $H_{\mathrm{K} 2}$ values as inputs (Fig. 2b). The $M_{\mathrm{s}}$ values of the continuous samples were measured using a vibrating sample magnetometer. The anisotropy constants were then obtained from the relationships $K_{1}=M_{\mathrm{s}} H_{\mathrm{K} 1}^{\text {eff }} / 2+$ $2 \pi M_{\mathrm{s}}^{2}$ and $K_{2}=M_{\mathrm{s}} H_{\mathrm{K} 2} / 4$. All measurements were performed at room temperature.

\section{Measurement of work function}

To measure the work functions of metals and $\mathrm{MgO}$, ultraviolet photoelectron spectroscopy (UPS) measurements were performed for separately prepared stacks of $\mathrm{Si}$ substrate (wet-oxidized)/X $(5 \mathrm{~nm})$ (including Co). The UPS measurements were performed using He I radiation $(h v=21.2 \mathrm{eV})$ from a gas-discharge lamp. The base pressure of the chamber was $2 \times 10^{-8}$ Torr. Prior to the measurement, Ar ion sputtering was performed to remove any native oxides formed during the exposure to air. The metallic films were sputtered repeatedly until the Fermi edge was observed. More details on the measurement of the work function and the photoemission spectra are provided in Supplementary Note 2.

\section{Results and discussion}

In Fig. $3 \mathrm{a}-\mathrm{c}, K_{1}$ is plotted as a function of the work function $(W)$, electronegativity $(\chi)$, and spin-orbit coupling constant $(\xi)$, all of which are taken from the literature $^{27-29}$. We choose these material parameters because of their potential correlation with the inversion asymmetry or Rashba effect at the Co/X interface ${ }^{30-32}$. To estimate the strength of the correlation, we calculated Pearson's $r$ for all the plots. Pearson's $r$ is close to $\pm 1(0)$ for a strong (weak) correlation. We obtain correlation coefficients of $0.82,0.63$, and 0.07 for the plots in Fig. 3a-c, respectively, indicating the strongest correlation between $K_{1}$ and $\Delta W\left(\equiv W_{\mathrm{X}}-W_{\mathrm{Co}}\right) . K_{1}$ also appears to be correlated with $X$ (Fig. 3b). This is expected because the difference in $X$ between two elements is proportional to the charge transfer ${ }^{33}$, which could be driven by the potential gradient at the $\mathrm{Co} / \mathrm{X}$ interface in our samples. We note that this correlation feature is in accordance with 


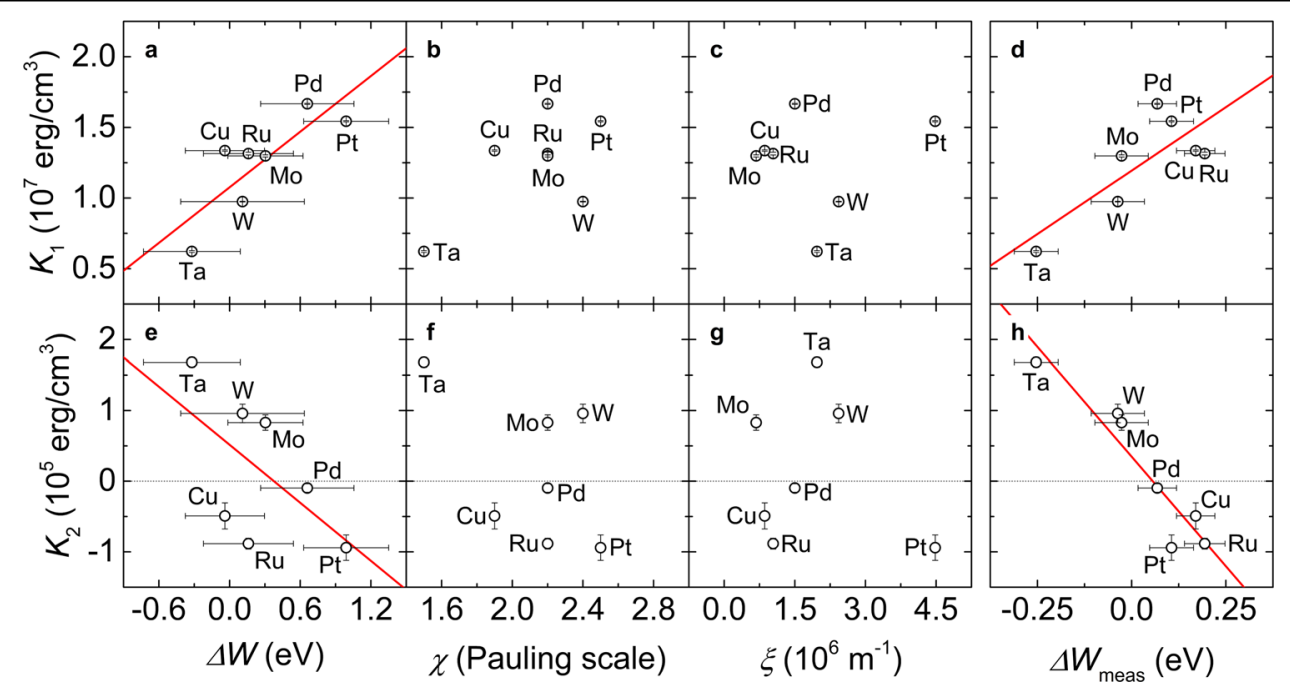

Fig. 3 Correlation of magnetic anisotropies with material parameters. a-d $K_{1}$ as a function of $\Delta W(\mathbf{a}), X(\mathbf{b}), \xi(\mathbf{c})$, and $\Delta W_{\text {meas }}(\mathbf{d})$ for $P t / C o / X$ stacks with various $X$ elements. $\mathbf{e}-\mathbf{h}$ Correlation results for $K_{2}$, similar to those shown in $(\mathbf{a}-\mathbf{d})$. The values of $\Delta W, X$, and $\xi$ were taken from the literature ${ }^{27-29}$, and those of $\Delta W_{\text {meas }}$ were obtained in this study via UPS measurements. The error bars of $K_{1}, K_{2}$, and $\Delta W_{\text {meas }}$ were obtained from three repeated measurements, whereas those of $\Delta W$ represent the standard deviations of reported values.

a recent experimental observation for the interfacial Dzyaloshinskii-Moriya interaction originating from inversion asymmetry ${ }^{27}$. We also plot $K_{1}$ as a function of $\triangle W$ measured for our samples by UPS (denoted as $\Delta W_{\text {meas }}$ ) (see Fig. 3d, Methods, and Supplementary Note 2 for details) and find a similar correlation between the two parameters $\left(K_{1}\right.$ and $\left.\Delta W_{\text {meas }}\right)$ with a correlation coefficient of 0.78 . This result shows that the inversion asymmetry at the interface plays an important role in the $K_{1}$ of $\mathrm{Pt} / \mathrm{Co} / \mathrm{X}$ heterostructures.

Figure $3 \mathrm{e}-\mathrm{h}$ shows the results for $K_{2}$, which are similar to those for $K_{1}$ shown in Fig. 3a-d. The correlation coefficients for $K_{2}$ are $-0.59,-0.51$, and -0.18 for literature values of $\Delta W, \chi$, and $\xi$, respectively. Similar to $K_{1}$, $K_{2}$ exhibits meaningful correlations with $\Delta W$ and $\chi$. The correlation coefficient of $K_{2}$ with $\Delta W_{\text {meas }}$ is substantially improved to -0.94 (Fig. $3 \mathrm{~h}$ ), suggesting a strong correlation. Importantly, $K_{2}$ changes its sign depending on the type of material $\mathrm{X}$ but still shows an almost linear correlation with $\Delta W_{\text {meas }}$. According to theoretical work, the Rashba spin-orbit coupling is known to be proportional to the surface potential (seen by electrons) and electron density distribution ${ }^{34}$. Since the surface potential of a metal-metal interface is equivalent to $\Delta W_{\text {meas }}$, the good correlation observed in Fig. 3h suggests that the inversion asymmetry is an intrinsic origin of $K_{2}$ in $\mathrm{Pt} / \mathrm{Co} / \mathrm{X}$ heterostructures. We call it intrinsic because this mechanism is distinct from the first (spatial fluctuations of $K_{1}{ }^{18}$ ) and second (interfacial PMA combined with a gradual weakening of the exchange energy along the thickness direction $^{19}$ ) mechanisms, which are extrinsic. Furthermore, our simple tight-binding model calculation with Rashba spin-orbit coupling supports this conclusion, as it shows that $K_{2}$ can have both positive and negative signs depending on the band filling even though $K_{1}$ is positive (i.e., PMA) (Supplementary Note 3). It is worth noting that the linear correlation, observed in Fig. 3d, h, persists even with the structural disordering taken into account (Supplementary Note 4). This indicates that the effect of the inversion asymmetry on the magnetic anisotropy can still be valid in intermixed thin-film structures ${ }^{17}$.

The linear correlation between $K_{2}$ and $\Delta W_{\text {meas }}$, however, appears to be somewhat unreasonable, considering that the magnetic anisotropy is known to originate from the second-order perturbative treatment of spin-orbit coupling near the Fermi level ${ }^{13,16}$. The following explanation can be given to understand the observed linear correlation. The effective electric field $\left(E_{0}\right)$ formed at the metal-metal interface will be large when the work-function difference between the two metals (e.g., Co and normal metal) is large. The use of a different normal metal will result in the modulation of $E_{0}, \mathrm{~d} E$, with a resultant $E_{0}$ value of $E_{0}+\mathrm{d} E$. Since the magnetic anisotropy is quadratic in the Rashba parameter (i.e., $\left.E_{0}+\mathrm{d} E\right)^{16}$, the variation of the magnetic anisotropy with a differing normal metal can be simply expressed as $\left(E_{0}+d E\right)^{2} \approx E_{0}^{2}+2 E_{0} d E$. It is then possible to explain our experimental results (Fig. $3 \mathrm{~d}$, h) that the modulation of the work function can give rise to the linear variation of the magnetic anisotropy.

The correlation result suggests that a large negative $\Delta W$ results in a large positive $K_{2}$, which is needed to form the easy-cone state. For experimental realization, we replace the metallic $\mathrm{X}$ layer with a $\mathrm{MgO}$ layer (see Supplementary Note 5). We choose MgO for the following two reasons. 

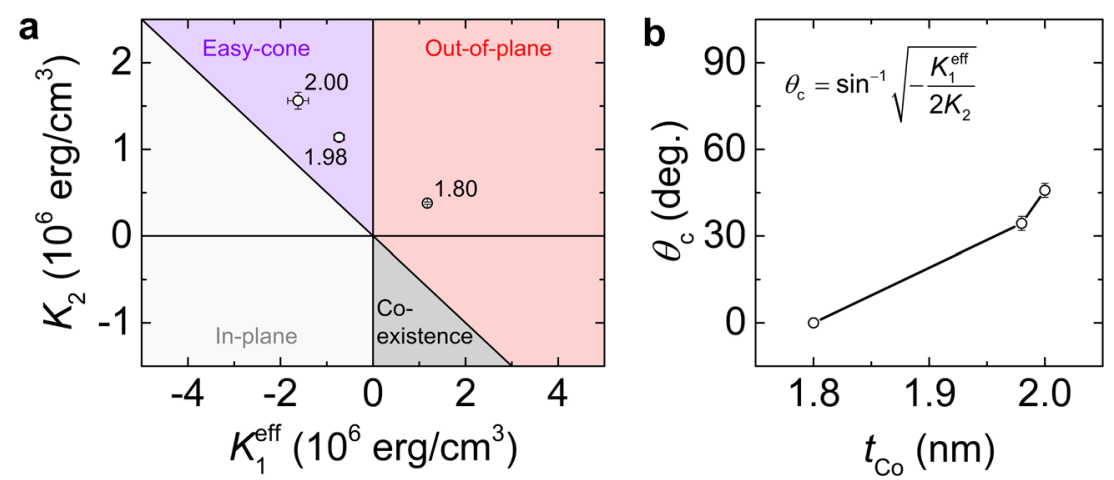

Fig. $4 \mathrm{Pt} / \mathrm{Co} / \mathrm{MgO}$ structure with easy-cone state. a Magnetic phase diagram in the $K_{1}^{\text {eff }}-K_{2}$ plane, with the $K_{1}^{\text {eff }}$ and $K_{2}$ values indicated at several $t_{C o}$ values in nanometers. $\mathbf{b}$ Plot of $\theta_{c} v s$. $t_{C o}$. The error bars of $K_{1}^{\text {eff }}, K_{2}$, and $\theta_{c}$ were obtained from three repeated measurements.

First, strong Rashba splitting was observed at metal-oxide interfaces ${ }^{31,32}$. Our $\Delta W_{\text {meas }}$ value at the $\mathrm{Co} / \mathrm{MgO}$ interface is consistent with this expectation: it is $-0.36 \mathrm{eV}$ (Supplementary Note 5), which is more negative than the value $(-0.25 \mathrm{eV})$ for the $\mathrm{Co} / \mathrm{Ta}$ interface, which exhibits the most negative $\Delta W_{\text {meas }}$ among all the metallic $\mathrm{Co} / \mathrm{X}$ interfaces. Second, $\mathrm{MgO}$ is widely adopted in various spintronic devices ${ }^{3}$. For a $\mathrm{Pt} / \mathrm{Co}(1.0 \mathrm{~nm}) / \mathrm{MgO}$ stack, we obtain a $K_{1}$ of $1.47 \times 10^{7} \mathrm{erg} / \mathrm{cm}^{3}$ and $K_{2}$ of $2.61 \times 10^{6} \mathrm{erg} / \mathrm{cm}^{3}$. Compared with the all-metallic structures, the $K_{2}$ of the $\mathrm{Pt} / \mathrm{Co} / \mathrm{MgO}$ structure is larger by an order of magnitude, which is in accordance with our conclusion in this work; the inversion asymmetry is an intrinsic origin of $K_{2}$. However, previously proposed mechanisms ${ }^{18-20}$ not considering the role of the inversion asymmetry are unable to explain the enhanced $K_{2}$ (see Supplementary Note 7 for details). Nonetheless, we note that the simple linear correlation between $K_{2}$ and $\Delta W_{\text {meas }}$ describes the enhanced $K_{2}$ of the $\mathrm{Pt} / \mathrm{Co} / \mathrm{MgO}$ structure only qualitatively, not quantitatively. Extrapolation of the linear line in Fig. 3 h gives a $K_{2}$ value of approximately $0.27 \times 10^{6} \mathrm{erg} / \mathrm{cm}^{3}$, which is significantly smaller than the measured value of $2.61 \times 10^{6} \mathrm{erg} / \mathrm{cm}^{3}$. A similar behavior is observed for $K_{1}$; in this case, the extrapolation gives a value of $0.55 \times 10^{7}$ $\mathrm{erg} / \mathrm{cm}^{3}$, which is approximately one third of a measured value of $1.47 \times 10^{7} \mathrm{erg} / \mathrm{cm}^{3}$. These deviations may indicate that $\Delta W_{\text {meas }}$ is not the sole factor determining the inversion asymmetry for a metal-oxide interface. A recent experimental work combined with a first-principles study found that the asymmetric charge-density distribution (or the charge transfer) at a metal-oxide interface has a larger effect on the Rashba splitting than the work-function difference (or the potential gradient) ${ }^{32}$.

This large and positive $K_{2}$ allows the easy-cone state to be formed in $\mathrm{Pt} / \mathrm{Co} / \mathrm{MgO}$ structures at $t_{\mathrm{Co}}$ near the spin reorientation transition ${ }^{1}$. The formation of the easy-cone state is validated by both vibrating sample magnetometry and AHE measurements (Supplementary Note 8). The
$K_{1}^{\text {eff }}$ and $K_{2}$ values for the $\mathrm{Pt} / \mathrm{Co}(1.8-2.0 \mathrm{~nm}) / \mathrm{MgO}$ structures are overlaid on a magnetic phase diagram (Fig. 4a). The cone angle $\left(\theta_{\mathrm{c}}\right)$ is estimated according to the relationship $\theta_{\mathrm{c}}=\sin ^{-1}\left(\sqrt{-K_{1}^{\text {eff }} / 2 K_{2}}\right)$. We find that $\theta_{\mathrm{c}}$ can be engineered by controlling $t_{\mathrm{Co}}$ (Fig. 4b), which is beneficial for device applications of the easy-cone state.

\section{Conclusion}

We investigated the origin of $K_{2}$ in $\mathrm{Pt} / \mathrm{Co} / \mathrm{X}$ heterostructures and found that inversion asymmetry plays an important role in $K_{2}$. Among the material parameters considered in this study, the work-function difference at the $\mathrm{Co} / \mathrm{X}$ interface shows the strongest correlation with both $K_{1}$ and $K_{2}$. Replacing the metallic X layer with $\mathrm{MgO}$, whose interface with Co has a strong inversion asymmetry, we obtain greatly enhanced $K_{2}$, allowing the easycone state. The intrinsic origin of $K_{2}$ revealed in this study will contribute to the control of its values and therefore allow various easy-cone states suitable for a wide variety of spintronic applications.

\section{Acknowledgements}

This research was supported by the Creative Materials Discovery Program through the National Research Foundation of Korea (No. 2015M3D1A1070465), the Samsung Electronics University R\&D program, and the KIST Institutional Program (Project Nos. 2V05750, 2E29410).

\section{Author details}

'Department of Materials Science and Engineering, Korea University, Seoul 02841, Korea. ${ }^{2}$ KU-KIST Graduate School of Converging Science and Technology, Korea University, Seoul 02841, Korea. ${ }^{3}$ Center for Spintronics, Korea Institute of Science and Technology, Seoul 02792, Korea

\section{Author contributions}

H.K.G. and S.H.L. planned and designed the experiment. H.K.G. prepared the samples and performed measurements. H.-J.P., K.-W.K., and K.-J.L. performed the theoretical analysis. All authors discussed the results and contributed to the manuscript. 


\section{Conflict of interest}

The authors declare that they have no conflict of interest.

\section{Publisher's note}

Springer Nature remains neutral with regard to jurisdictional claims in published maps and institutional affiliations.

Supplementary information is available for this paper at https://doi.org/ 10.1038/s41427-020-0205-z.

\section{Received: 6 June 2019 Revised: 9 December 2019 Accepted: 7 January} 2020.

Published online: 6 March 2020

\section{References}

1. Millev, Y. \& Kirschner, J. Reorientation transitions in ultrathin ferromagnetic films by thickness- and temperature-driven anisotropy flows. Phys. Rev. B 54, 4137-4145 (1996).

2. Dieny, B. \& Chshiev, M. Perpendicular magnetic anisotropy at transition metal/ oxide interfaces and applications. Rev. Mod. Phys. 89, 025008 (2017).

3. Ikeda, S. et al. A perpendicular-anisotropy CoFeB-MgO magnetic tunnel junction. Nat. Mater. 9, 721-724 (2010).

4. Matsumoto, R., Arai, H., Yuasa, S. \& Imamura, H. Spin-transfer-torque switching in a spin-valve nanopillar with a conically magnetized free layer. Appl. Phys. Express 8, 063007 (2015).

5. Strelkov, N. et al. Stability phase diagram of a perpendicular magnetic tunnel junction in noncollinear geometry. Phys. Rev. B 95, 184409 (2017).

6. Bultynck, $\mathrm{O}$. et al. Instant-on spin torque in noncollinear magnetic tunnel junctions. Phys. Rev. Appl. 10, 054028 (2018).

7. Jang, P.-H., Lee, S.-W. \& Lee, K.-J. Spin-transfer-torque-induced zero-field microwave oscillator using a magnetic easy cone state. Curr. Appl. Phys. 16, 1550-1553 (2016)

8. Sonin, E. B. Spin currents and spin superfluidity. Adv. Phys. 59, 181-255 (2010).

9. Kim, S. K. \& Tserkovnyak, Y. Interaction between a domain wall and spin supercurrent in easy-cone magnets. Phys. Rev. B 94, 220404 (2016).

10. Jang, P.-H., Oh, S.-H., Kim, S. K. \& Lee, K-J. Domain wall dynamics in easy-cone magnets. Phys. Rev. B 99, 024424 (2019).

11. Timopheev, A. A. et al. Inhomogeneous free layer in perpendicular magnetic tunnel junctions and its impact on the effective anisotropies and spin transfer torque switching efficiency. Phys. Rev. B 96, 14412 (2017).

12. Teixeira, B. M. S. et al. Ion irradiation-induced easy-cone anisotropy in double$\mathrm{MgO}$ free layers for perpendicular magnetic tunnel junctions. Appl. Phys. Lett. 112, 202403 (2018).

13. Bruno, P. Tight-binding approach to the orbital magnetic moment and magnetocrystalline anisotropy of transition-metal monolayers. Phys. Rev. B 39, 865(R) (1989).

14. Nakamura, K. et al. Giant modification of the magnetocrystalline anisotropy in transition-metal monolayers by an external electric field. Phys. Rev. Lett. 102, 187201 (2009).
15. Barnes, S. E., leda, J. \& Maekawa, S. Rashba spin-orbit anisotropy and the electric field control of magnetism. Sci. Rep. 4, 4105 (2014).

16. Kim, K.-W., Lee, K.J., Lee, H.-W. \& Stiles, M. D. Perpendicular magnetic anisotropy of two-dimensional Rashba ferromagnets. Phys. Rev. B 94, 184402 (2016).

17. Pradipto, A.M. et al. Enhanced perpendicular magnetocrystalline anisotropy energy in an artificial magnetic material with bulk spin-momentum coupling. Phys. Rev. B 99, 180410(R) (2019).

18. Dieny, B. \& Vedyayev, A. Crossover from easy-plane to perpendicular anisotropy in magnetic thin films: canted anisotropy due to partial coverage or interfacial roughness. Europhys. Lett. 25, 723-728 (1994).

19. Sun, J. Z. Consequences of an interface-concentrated perpendicular magnetic anisotropy in ultrathin CoFeB films used in magnetic tunnel junctions. Phys. Rev. B 91, 174429 (2015).

20. Tomáš, l., Murtinová, L. \& Kaczér, J. Easy magnetization axes in materials with combined cubic and uniaxial anisotropies. Phys. Stat. Sol. A 75, 121-127 (1983).

21. Fritzsche, H., Kohlhepp, J., Elmers, H. J. \& Gradmann, U. Angular dependence of perpendicular magnetic surface anisotropy and the spin-reorientation transition. Phys. Rev. B 49, 15665 (1994).

22. Oepen, H. P., Speckmann, M., Millev, Y. \& Kirschner, J. Unified approach to thickness-driven magnetic reorientation transitions. Phys. Rev. B 55, 2752 (1997).

23. Rodmacq, B., Manchon, A., Ducruet, C., Auffret, S. \& Dieny, B. Influence of thermal annealing on the perpendicular magnetic anisotropy of $\mathrm{Pt} / \mathrm{Co} / \mathrm{AlOx}$ trilayers. Phys. Rev. B 79, 024423 (2009).

24. Yang, Y. et al. Unveiling the role of Co-O-Mg bond in magnetic anisotropy of $\mathrm{Pt} / \mathrm{Co} / \mathrm{MgO}$ using atomically controlled deposition and in situ electrical measurement. Phys. Rev. B 95, 094417 (2017).

25. Gweon, H. K., Yun, S. J. \& Lim, S. H. A very large perpendicular magnetic anisotropy in $\mathrm{Pt} / \mathrm{Co} / \mathrm{MgO}$ trilayers fabricated by controlling the $\mathrm{MgO}$ sputtering power and its thickness. Sci. Rep. 8, 1266 (2018).

26. Sucksmith, W. \& Thompson, J. E. The magnetic anisotropy of cobalt. Proc. $R$. Soc. A 225, 362 (1954)

27. Park, Y.-K. et al. Experimental observation of the correlation between the interfacial Dzyaloshinskii-Moriya interaction and work function in metallic magnetic trilayers. NPG Asia Mater. 10, 995-1001 (2018).

28. Huheey, J. E., Keiter, E. A. \& Keiter, R. L. Inorganic Chemistry. 4th edn, (HarperCollins College Publishers, New York, NY, USA, 1993).

29. Montalti, M., Credi, A., Prodi, L. \& Gandofi, M. T., Handbook of Photochemistry. 3rd edn (CRC Press, Boca Raton, FL, 2006).

30. Petersen, L. \& Hedegård, P. A simple tight-binding model of spin-orbit splitting of sp-derived surface states. Surf. Sci. 459, 49-56 (2000).

31. Krupin, O. et al. Rashba effect at magnetic metal surfaces. Phys. Rev. B 71 201403(R) (2005).

32. Tsai, $\mathrm{H}$. et al. Clear variation of spin splitting by changing electron distribution at non-magnetic metal/ $\mathrm{Bi}_{2} \mathrm{O}_{3}$ interface. Sci. Rep. 8, 5564 (2018).

33. Miedema, A. R. The electronegativity parameter for transition metals: Heat of formation and charge transfer in alloys. J. Less-Common Met. 32, 117 (1973).

34. Nagano, M., Kodama, A., Shishidou, T. \& Oguchi, T. A first-principles study on the Rashba effect in surface systems. J. Phys.: Condens. Matter 21, 064239 (2009). 